\title{
TREINAMENTO COM PESOS E PROMOÇÃO DA SAÚDE EM ADULTOS: UMA REVISÃO SISTEMÁTICA DA LITERATURA NACIONAL ACERCA DOS MÉTODOS EMPREGADOS EM ESTUDOS CIENTÍFICOS
}

\author{
Lourival Cecotti de Andrade, Rômulo Araújo Fernandes \\ Curso de Educação Física da UNOESTE, Presidente Prudente, SP.
}

\section{RESUMO}

A prática do treinamento resistido (TR) tem sido estimulada por importantes sociedades relacionadas à medicina do esporte e prevenção/tratamento de algumas doenças de ordem musculoesqueléticas e, assim, sua prática para o tratamento de doenças crônicas tem sido discutida. Porém, há limitações severas no delineamento experimental de muitos estudos e tal limitação cria vieses em sua aplicação para tais fins. $O$ objetivo deste estudo foi analisar, utilizando um modelo de revisão sistemática da literatura nacional, a qualidade metodológica dos artigos científicos empregados no treinamento com pesos em adultos e, dessa forma, identificar possíveis lacunas na literatura nacional. A revisão sistemática da literatura foi realizada em uma base de dados eletrônica. A busca eletrônica foi realiza na Scientific Electronic Library Online (SCIELO). Dentre os artigos detectados, critérios de inclusão foram estabelecidos: (i) estudo original envolvendo a prática do treinamento com pesos, (ii) estudo realizado no Brasil, (iii) não ser estudo de revisão, (iv) não envolver outros grupos etários, caso de crianças e adolescentes. A análise estatística dos dados foi composta pelo teste qui-quadrado para comparar proporções e teste exato do Fisher. Dentre os estudos que analisaram desfechos relacionados à saúde, $75 \%$ utilizaram grupo controle, ao passo que apenas $26,7 \%$ dos estudos que não analisaram saúde utilizaram grupo controle. Apenas $47,8 \%$ dos estudos utilizaram o teste de 1RM para prescrever intensidade. Dos 23 artigos analisados apenas $30,4 \%$ utilizou a familiarização previamente ao protocolo de treinamento. Há mais trabalhos científicos com amostras do sexo masculino. Por fim, nossos achados permitem concluir que há significativa ausência de critério na condução dos estudos nacionais envolvendo TR. Além disso, existe pouca informação nacional sobre a aplicação do TR visando melhoras na saúde e que tal ausência é mais sentida em grupos etários específicos, caso de crianças e adolescentes.

Palavras-chave: Treinamento com pesos, musculação, treinamento resistido, adultos, Brasil.

\section{WEIGHT TRAINING AND ADULTS' HEALTH PROMOTION: A SYSTEMATIC REVIEW ABOUT METHODS USED IN SCIENTIFIC STUDIES}

\begin{abstract}
The practice of resistance training (RT) has been stimulated by important societies related to sports medicine and prevention/treatment of some diseases of musculoskeletal order and thus their practice for the treatment of chronic diseases has been discussed. However, there are severe limitations in the experimental design of many studies, this limitation creates bias in its application for such purposes. The aim of this study was to analyze, using a model of systematic review of national literature, the methodological quality of the papers used in weight training in adults and thus identify possible gaps in the national literature. A systematic literature review was conducted in an electronic database. The electronic search was performed in the Scientific Electronic Library Online (SciELO). Among the detected items, inclusion criteria were established: (i) original study involving the practice of weight training, (ii) study conducted in Brazil, (iii) is not a review article, (iv) does not involve other age groups, as children and adolescents. The statistical analysis was made using the chi-square test to compare proportions and the Fisher exact test. Among the studies that examined health-related outcomes, $75 \%$ used a control group, while only $26.7 \%$ of the studies did not analyze health used a control group. Only $47.8 \%$ of the studies used the 1 RM test to prescribe intensity. Of the 23 articles analyzed only $30.4 \%$ used prior to the familiarization training protocol. There are more scientific studies male samples. Finally, our findings support the conclusion that there is a significant lack of discretion in the conduct of national studies involving RT. In addition, there is little national information on the RT to improve health and that this absence is most felt in specific age groups such as children and adolescents.
\end{abstract}

Keywords: Weight training, bodybuilding, resistance training, adult, Brazil. 


\section{INTRODUÇÃO}

A prática do treinamento com resistido (TR) tem sido estimulada por importantes sociedades relacionadas à medicina do esporte para prevenção/tratamento de algumas doenças de ordem musculoesqueléticas. Da mesma forma, o tema tem sido amplamente discutido por outras sociedades relacionadas ao tratamento de algumas doenças, caso da obesidade, hipertensão arterial, diabetes mellitus e dislipidemia.

Nesse sentido, com base no grande destaque assumido pelo assunto, é comum o seu emprego na prática profissional do educador físico, seja em áreas relacionadas ao treinamento esportivo (futebol, atletismo, lutas etc), seja em áreas relacionadas à promoção da saúde (academias, clubes privados etc). Por outro lado, em decorrência da dificuldade de se trabalhar com esta modalidade de exercício (equipamentos específicos, alta taxa de desistência etc), sabe-se que há limitações severas no delineamento experimental de muitos estudos (poucos estudos com mulheres, períodos variados de intervenção, protocolos pouco claros, ausência de grupos controles etc). Tal limitação é tão evidente que importantes sociedades têm indicado a inexistência de evidências científicas para indicar o treinamento com pesos como forma de prevenção/tratamento de desfechos como obesidade, dislipidemia e hipertensão arterial.

O pano de fundo acima exposto cria um problema, dado o grande uso do TR em intervenções visando melhorias em tais patologias. Esta revisão sistemática da literatura nacional acerca do assunto buscou identificar possíveis lacunas no que se refere à aplicação do TR na prevenção e tratamento de algumas patologias. Partimos da hipótese inicial de que há poucos estudos explorando os possíveis benefícios do treinamento com pesos na prevenção/tratamento de algumas patologias, bem como, avançamos com a segunda hipótese de que há limitações severas no delineamento experimental de tais estudos.

O objetivo deste estudo foi analisar, utilizando um modelo de revisão sistemática da literatura nacional, os métodos empregados no treinamento com pesos em adultos e, dessa forma, identificar possíveis lacunas na literatura nacional.

\section{MÉTODOS}

A revisão sistemática da literatura foi realizada em uma base de dados eletrônica, não foi feita busca manual por documentos, bem como, não se entrou em contato com autores, devido ao fato da base selecionada disponibilizar gratuitamente todos os artigos. Assim, por ser a maior base nacional de periódicos científicos, a busca eletrônica foi realiza na Scientific Electronic Library Online (SCIELO) e não foi feita busca em outras revistas da área de Educação Física não indexadas na base SCIELO.

Foram utilizadas as seguintes palavraschave: treinamento com pesos, treinamento de força, musculação, treinamento resistido. Não foi feita busca de artigos utilizando os descritores correspondentes em língua inglesa.

Dentre os artigos detectados, uma análise inicial foi realizada com bases nos títulos e nos resumos dos mesmos para alcançar a certeza de que os artigos se enquadravam nos critérios de inclusão estabelecidos: (i) estudo original envolvendo a prática do treinamento com pesos, (ii) estudo realizado no Brasil, (iii) não ser estudo de revisão, (iv) não envolver outros grupos etários, caso de crianças e adolescentes. Após a leitura do título, a análise do resumo foi feita, caso houvesse dúvida no cumprimento dos critérios de inclusão. Os artigos selecionados foram obtidos na integra e posteriormente examinados, buscando 0 arquivamento das variáveis independentes (presença de grupo controle, sexo 
dos participantes, tempo de intervenção do protocolo [se houve], familiarização prévia ao programa de exercícios proposto, utilização do teste de uma repetição máxima [1RM]).

Uma planilha inicial foi preenchida com as informações metodológicas relevantes para este estudo (acima citadas), bem como, os artigos que não cumpriram os critérios de inclusão foram excluídos das análises principais (Tabelas 1 e 2).

A análise estatística dos dados foi composta pelo teste qui-quadrado para comparar proporções, bem como, em decorrência do baixo número de casos observados (estudos encontrados [n=23]), foi empregado o teste exato do Fisher para analisar possíveis associações. Todas as análises foram efetuadas em software estatístico e o nível de significância foi pré-fixado em $p<0,05$.

\section{RESULTADOS}

Dentre os 37 artigos encontrados em nossa busca de dados, todos foram encontrados na Revista Brasileira de Medicina do Esporte, bem como, sete eram de revisão e, assim, foram excluídos das análises principais. Dentre estes sete trabalhos de revisão, os desfechos analisados foram variados, incluindo: perfil lipídico, densidade mineral óssea, pressão arterial, etc. Além disso, um estudo analisou a confiabilidade do teste de 1RM (Tabela 1).

Tabela 1. Estudos de revisão excluídos.

\begin{tabular}{|c|c|c|c|}
\hline Autor & Revista & Ano & Tema \\
\hline Cadone e col. & RBME & 2008 & Respostas da testosterona e cortisol ao TR \\
\hline da Silva e Maranhão & RBME & 2010 & Efeitos do TR na lipoproteína de baixa densidade \\
\hline da Silva1 e Farinatti & RBME & 2007 & $\begin{array}{l}\text { TR sobre a força muscular de idosos: relação dose- } \\
\text { resposta }\end{array}$ \\
\hline Dourado e Godoy & RBME & 2004 & $\begin{array}{l}\text { Recondicionamento muscular na DPOC: principais } \\
\text { intervenções e novas tendências. }\end{array}$ \\
\hline Cadore e col. & RBME & 2005 & $\begin{array}{l}\text { Efeitos da atividade física na densidade mineral óssea e } \\
\text { na remodelação do tecido ósseo. }\end{array}$ \\
\hline Polito e Farinatti & RBME & 2003 & A medida da pressão arterial em exercícios resistido \\
\hline Pereira1 e Gomes & RBME & 2003 & $\begin{array}{l}\text { Teste de força e resistência muscular: confiabilidade e } \\
\text { predição de uma repetição máxima. }\end{array}$ \\
\hline
\end{tabular}

Outros sete estudos foram excluídos por envolverem idosos ( $n=5)$ ou crianças $(n=2)$. Entre os trabalhos envolvendo crianças, os temas foram relacionados ao destreino e uso do teste de 1RM. Não foram encontrados estudos analisando seus possíveis benefícios na saúde de populações pediátricas. Dentre os idosos, a maioria abordou o seu efeito no ganho de força, porém, abordouse também o seu efeito sobre a densidade mineral óssea e pressão arterial (Tabela 2).

A Figura 1 apresenta o número de artigos publicados ao longo dos anos de 2001 e 2010 (Painel A), bem como, os tipos de artigos publicados (Painel B).
Foi possível evidenciar sensível aumento no número de artigos publicados, porém, com alguma queda nos últimos anos. Da mesma forma, a grande maioria dos trabalhos publicados foi original envolvendo adultos $(p=0,001)$. Poucos trabalhos envolveram crianças e idosos.

Nossos resultados evidenciaram que, dentre os estudos selecionados para nossa metaanalise, embora com significância marginal $(p=0,062)$, há um número maior de estudos conduzidos com homens do que com mulheres (Figura 2). 
Tabela 2. Estudos envolvendo idosos e crianças que foram excluídos.

\begin{tabular}{|c|c|c|c|}
\hline $\begin{array}{l}\text { Estudos com crianças } \\
\text { Autor }\end{array}$ & Revista & Ano & Tema \\
\hline Fontoura e Meyer & RBME & 2004 & $\begin{array}{l}\text { O efeito do destreinamento de força muscular em } \\
\text { meninos pré-púberes }\end{array}$ \\
\hline Gurjão e col. & RBME & 2005 & $\begin{array}{l}\text { Variação da força muscular em testes repetitivos de 1- } \\
\text { RM em crianças pré-púberes }\end{array}$ \\
\hline \multicolumn{4}{|l|}{ Estudos com idosos } \\
\hline Jambassi filho e col. & RBME & 2010 & $\begin{array}{l}\text { Diferentes intervalos de recuperação entre as séries, na } \\
\text { força muscular em mulheres idosas treinadas }\end{array}$ \\
\hline Raso e col. & RBME & 2001 & $\begin{array}{l}\text { A força muscular de mulheres idosas cresce } \\
\text { principalmente após oito semanas de interrupção de um } \\
\text { programa de exercícios com pesos livres }\end{array}$ \\
\hline Battaglini e col. & RBME & 2006 & $\begin{array}{l}\text { Efeitos do TR na força muscular e níveis de fadiga em } \\
\text { pacientes com câncer de mama }\end{array}$ \\
\hline Kemper e col. & RBME & 2009 & $\begin{array}{l}\text { Efeitos da natação e do TR na densidade mineral óssea } \\
\text { de mulheres idosas }\end{array}$ \\
\hline Mediano e col. & RBME & 2005 & $\begin{array}{l}\text { Comportamento subagudo da pressão arterial após o } \\
\text { treinamento de força em hipertensos controlados }\end{array}$ \\
\hline
\end{tabular}

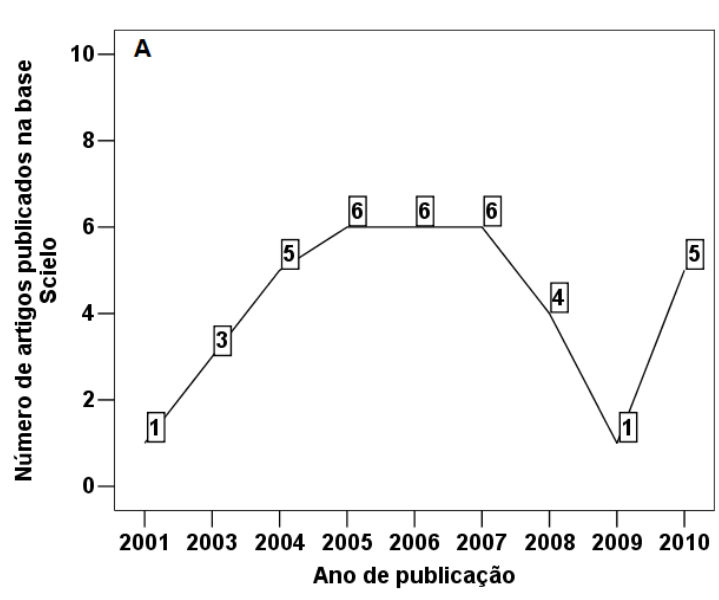

Figura 1. Número de artigos publicados na base entre aos anos de 2001 e 2010 (Painel A) e tipos de artigos publicados (Painel $B$ ).

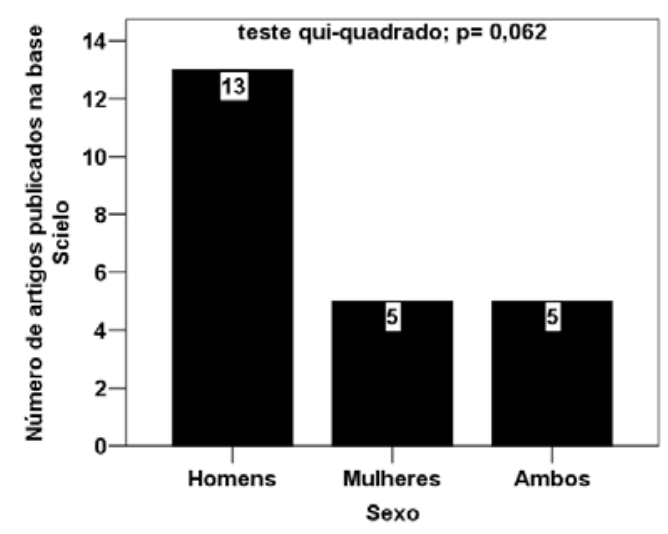

Figura 2. Número de artigos publicados segundo o sexo dos participantes.

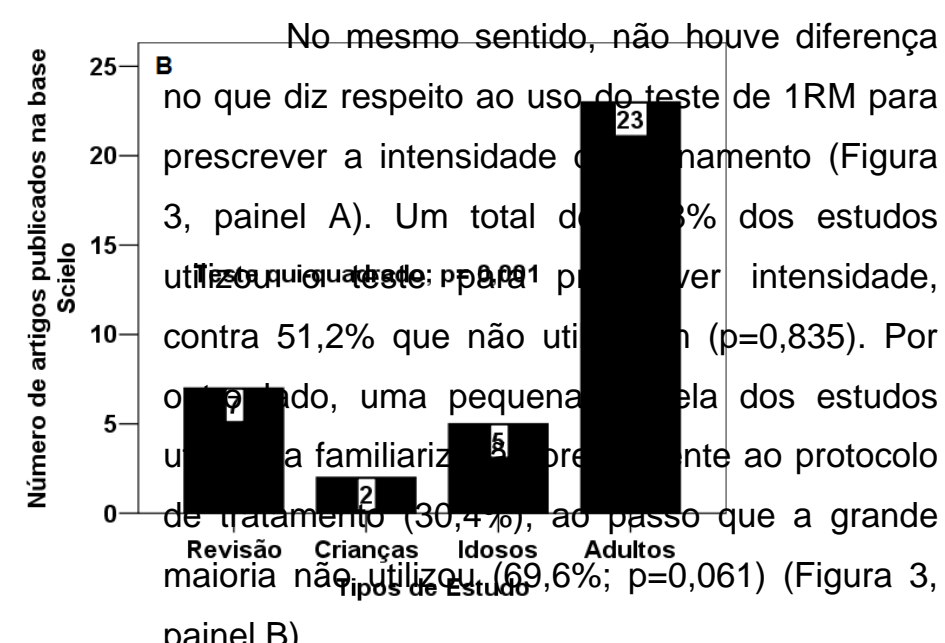

painel B).

Dentre todos os 37 estudos encontrados, quando analisados aqueles que abordavam o uso do TR para o tratamento de alguma patologia da síndrome metabólica e osteoporose, apenas $27 \%$ $(\mathrm{n}=10)$ abordavam o assunto. Assim, houve um número muito maior de estudos analisando outros desfechos ( $n=27 ; 73 \% ; p=0,005)$, tais como, TR e suplementação, efeito em alguns hormônios e ganho de massa muscular. Dentre os 23 estudos que cumpriram os critérios de inclusão e que analisaram desfechos relacionados à saúde, 75\% utilizaram grupo controle, ao passo que apenas 26,7\% dos estudos que não analisaram saúde utilizaram grupo controle $(p=0,103)$. 

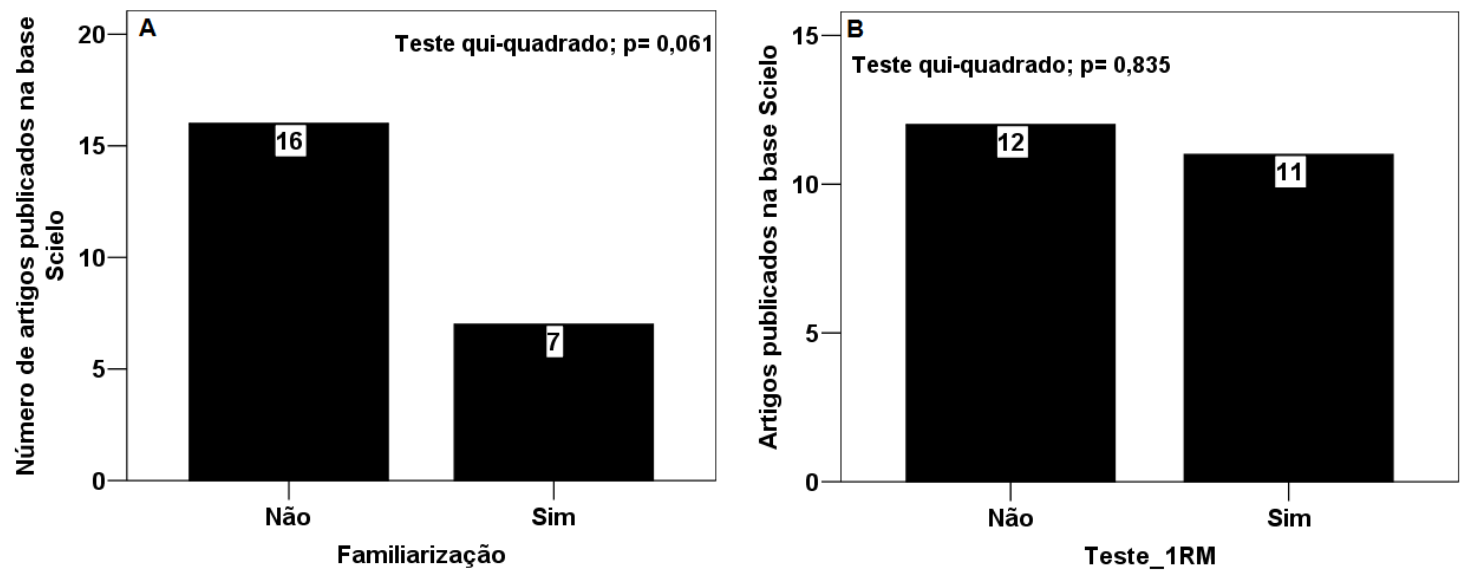

Figura 3. Número de estudos que utilizaram familiarização (Painel A) e teste de 1RM (Painel B).

\section{DISCUSSÃO}

Com este estudo foi possível evidenciar que há mais trabalhos científicos com amostras do sexo masculino e isso nos leva a questionar a inferência de tais resultados para o sexo feminino. Alterações na proporção dos tipos de fibras musculares entre homens e mulheres também podem interferir na capacidade de resistir à fadiga, uma vez que as mulheres possuem menor diferença na proporção entre fibras do tipo I e do tipo II (FLECK \& KRAEMER, 2007). Quando comparadas com os homens, o que pode favorecer a capacidade de resistir a esforços submáximos por períodos mais prolongados, homens e mulheres apresenta comportamentos relativamente diferentes em séries múltiplas de exercício com pesos. Desta forma, encontramos apenas 10 artigos científicos referentes ao TR, que tenham avaliado mulheres. Tal resultado, se considerado o grande período de tempo em que foi compreendido o levantamento (10 anos), constitui uma quantidade relativamente baixa de informação.

Também foi possível verificar que a maioria dos trabalhos científicos conduzidos envolveu grupos compostos por adultos; e aqueles que envolveram crianças e idosos foram poucos. Nesse sentido, uma vez que uma grande fatia do mercado atende essa população e que entidades importantes têm recomendado a prática do TR para essas populações, tal quadro constitui uma limitação. Além disso, podemos dizer que não há consistência na literatura nacional no que se refere ao TR em idosos e crianças, principalmente no que diz respeito a populações pediátricas.

Dentre os estudos que analisaram desfechos relacionados à saúde, $75 \%$ utilizaram grupo controle, ao passo que apenas $26,7 \%$ dos estudos que não analisaram saúde utilizaram grupo controle. A grande minoria dos estudos encontrados na base de dados SCIELO foram referente à promoção da saúde $(n=4)$, porém, grande parte deles utilizou grupo controle, deixando o resultado do protocolo de TR mais consistente. Por outro lado, artigos que não analisaram saúde, a grande maioria deles não utilizou grupo controle deixando dúvidas sobre os resultados obtidos. Desta forma, podemos dizer que há baixa qualidade metodológica nos estudos analisados, principalmente naqueles relacionados ao treinamento visando ganho de força ou hipertrofia.

Um total de $47,8 \%$ dos estudos utilizou o teste de 1RM para prescrever intensidade, ao passo que $51,2 \%$ que não utilizaram. Por outro 
lado, uma pequena parcela dos estudos utilizou a familiarização previamente ao protocolo de tratamento $(30,4 \%)$. Desta forma podemos dizer que de todos os 23 artigos analisados apenas quatro artigos foram realizados com consistência mínima nos métodos. Fazer teste de 1RM sem familiarização torna o estudo pouco claro devido o nível de coordenação motora não estarem igualadas isso torna o teste obscuro, ou seja, sem resultados confiáveis.

A principal limitação do estudo reside no fato de apenas a base SCIELO ter sido consultada. Sabe-se que outros estudos sobre o tema, inclusive nacionais, podem ter sido publicados em revista internacionais não indexadas no SCIELO. Assim, futuros estudos deveriam envolver outras bases de dados.

Por fim, nossos achados permitem concluir que há significativa ausência de critério na condução dos estudos nacionais envolvendo TR, uma vez que cuidados metodológicos básicos não são seguidos. Além disso, existe pouca informação nacional sobre a aplicação do TR visando melhoras na saúde e que tal ausência é mais sentida em grupos etários específicos, caso de crianças e adolescentes.

\section{CONFLITO DE INTERESSE}

Os autores declaram não haver qualquer potencial conflito de interesse que possa interferir na imparcialidade deste trabalho científico.

\section{REFERÊNCIAS}

Altimari LR et al. Efeitos da Suplementação Prolongada de Creatina Mono-Hidratada sobre o Desempenho Anaeróbio de Adultos Jovens Treinados. Rev Bras Med Esporte. 2010;16(3):186-90. http://dx.doi.org/10.1590/S1517$\underline{86922010000300006}$

Assunção WD et al. Respostas cardiovasculares agudas no treinamento de força conduzido em exercícios para grandes e pequenos grupamentos musculares. Rev Bras Med Esporte.
2007;13(2):118-22.

http://dx.doi.org/10.1590/S151786922007000200010

Battaglini $C$ et al. Efeitos do treinamento de resistência na força muscular e níveis de fadiga em pacientes com câncer de mama. Rev Bras Med Esporte. 2006;12(3):153-8.

Cadone EL et al. Fatores Relacionados com as Respostas da Testosterona e do Cortisol ao Treinamento de Forca. Rev Bras Med Esporte. 2008;14(1):74-8. http://dx.doi.org/10.1590/S151786922008000100014

Cadore EL et al. Efeitos da atividade física na densidade mineral óssea e na remodelação do tecido ósseo. Rev Bras Med Esporte. 2005;11(6):373-8.

http://dx.doi.org/10.1590/S151786922005000600013

Cyrino ES et al. Comportamento da flexibilidade após 10 semanas de treinamento com pesos. Rev Bras Med Esporte. 2004;10(4):233-7. http://dx.doi.org/10.1590/S151786922004000400001

Dias RMR et al. Impacto de oito semanas de treinamento com pesos sobre a força muscular de homens e mulheres. Rev Bras Med Esporte. 2005;11(4):224-8.

http://dx.doi.org/10.1590/S151786922005000400004

Dourado VZ, Godoy I. Recondicionamento muscular na DPOC: principais intervenções e novas tendências. Rev Bras Med Esporte. 2004;10(4):331-4.

Fleck SJ, Kraemer WL. Fundamentos do treinamento de força muscular. 3ed. Porto Alegre: Artmed; 2007.

Gentil $P$ et al. Efeitos agudos de vários métodos de treinamento de força no lactato sanguíneo e características de cargas em homens treinados recreacionalmente. Rev Bras Med Esporte. 2006;12(6):303-7.

Gerage AM et al. Efeito de 16 semanas de treinamento com pesos sobre a pressão arterial em mulheres normotensas e não-treinadas. Rev Bras Med Esporte. 2007;13(6):361-5.

Gomes RV, Aoki MS. Suplementação de creatina anula o efeito adverso do exercício de endurance sobre o subseqüente desempenho de força. Rev Bras Med Esporte. 2005;11(2):131-4. http://dx.doi.org/10.1590/S151786922005000200007 
Gurjão ALD et al. Variação da força muscular em testes repetitivos de 1-RM em crianças prépúberes. Rev Bras Med Esporte. 2005;11(6):31924.

Júnior TPS et al. Suplementação de creatina e treinamento de força: alterações na resultante de força máxima dinâmica e variáveis antropométricas em universitários submetidos a oito semanas de treinamento de força (hipertrofia). Rev Bras Med Esporte. 2007;13(5):303-9.

Kemper $\mathrm{C}$ et al. Efeitos da natação e do treinamento resistido na densidade mineral ossea de mulheres idosas. Rev Bras Med Esporte. 2009;15(1):10-13.

http://dx.doi.org/10.1590/S151786922009000100002

Lima FV et al. Análise de dois treinamentos com diferentes durações de pausa entre séries baseadas em normativas previstas para a hipertrofia muscular em indivíduos treinados. Rev Bras Med Esporte. 2006;12(4):175-8.

Maesta $\mathrm{N}$ et al. Efeito da Oferta Dietética de Proteína Sobre o Ganho Muscular, Balanço Nitrogenado e Cinética da 15N-Glicina de Atletas em Treinamento de Musculação. Rev Bras Med Esporte. 2008;3(3):215-9.

Martins B et al. Efeitos do Intervalo de Recuperacao Entre Series de Exercicios Resistidos no Hormônio do Crescimento em Mulheres Jovens. Rev Bras Med Esporte. 2008;14(3):171-5.

http://dx.doi.org/10.1590/S151786922008000300002

Monteiro W. Manipulação na ordem dos exercícios e sua influência sobre número de repetições e percepção subjetiva de esforço em mulheres treinadas. Rev Bras Med Esporte. 2005;11(2):146-50.

Monteiro WD et al. Respostas Cardiovasculares Agudas ao Exercício de Força Realizado em Três Diferentes Formas de Execução. Rev Bras Med Esporte. 2008;14(2):94-8.

http://dx.doi.org/10.1590/S151786922008000200002

Neto JF et al. Alterações na composição corporal decorrentes de um treinamento de musculação em portadores de Síndrome de Down. Rev Bras Med Esporte. 2010;16(1):9-12.

Oliveira JC et al. Identificação do limiar de lactato e limiar glicêmico em exercícios resistidos. Rev Bras Med Esporte. 2006;12(6):333-7.
Oliveira PV et al. Correlação entre a suplementação de proteína e carboidrato e variáveis antropométricas e de força em indivíduos submetidos a um programa de treinamento com pesos. Rev Bras Med Esporte. 2006;12(1):51-5. http://dx.doi.org/10.1590/S1517$\underline{86922006000100010}$

Pereira MIR, Gomes PSC. Efeito do treinamento contra-resistência isotônico com duas velocidades de movimento sobre os ganhos de força. Rev Bras Med Esporte. 2007;13(2):91-6.

Polito MD et al. Efeito hipotensivo do exercício de força realizado em intensidades diferentes e mesmo volume de trabalho. Rev Bras Med Esporte. 2003;9(2):69-73.

Polito MD et al. Respostas cardiovasculares agudas na extensão do joelho realizada em diferentes formas de execução. Rev Bras Med Esporte. 2004;10(3):173-6.

Polito MD. Efeitos de 12 Semanas de Treinamento Com Pesos Sobre a Força Muscular, Composição Corporal e Triglicérides em Homens Sedentários. Rev Bras Med Esporte. 2010;16(1):29-32.

http://dx.doi.org/10.1590/S151786922010000100005

Pereira MIR, Gomes PSC. Testes de força e resistência muscular: confiabilidade e predição de uma repetição máxima - Revisão e novas evidências. Rev Bras Med Esporte. 2003;9(5):325-35.

Polito MD, Farinatti PTV. Considerações sobre a medida da pressão arterial em exercícios contraresistência. Rev Bras Med Esporte. 2003;9(1):2533.

Raso $\mathrm{V}$ et al. A força muscular de mulheres idosas decresce principalmente após oito semanas de interrupção de um programa de exercícios com pesos livres. Rev Bras Med Esporte. 2001;7(6):177-86.

Salvador EP et al. Comparação entre o desempenho motor de homens e mulheres em séries múltiplas de exercícios com pesos. Rev Bras Med Esporte. 2005;11(5):257-61.

Santos KB et al. Função muscular esquelética e composição corporal de pacientes com hipertireoidismo submetidos ao treinamento contra resistência. Rev Bras Med Esporte. 2001;7(1):18-23.

Silva JL et al. Efeitos do Treinamento Resistido na Lipoproteína de Baixa Densidade. Rev Bras Med Esporte. 2010;16(1):71-6. 
http://dx.doi.org/10.1590/S1517-

$\underline{86922010000100014}$

Silva NL, Farinatti PTV. Influência de variáveis do treinamento contra-resistência sobre a força muscular de idosos: uma revisão sistemática com ênfase nas relações dose-resposta. Rev Bras Med Esporte. 2007;13(1):60-6.

Uchida MC et al. Alteração da relação testosterona: cortisol induzida pelo treinamento de força em mulheres. Rev Bras Med Esporte. 2004;10(3):165-8.

http://dx.doi.org/10.1590/S1517-

$\underline{86922004000300005}$

Uchida MC et al. Efeito de diferentes protocolos de treinamento de força sobre parâmetros morfofuncionais, hormonais e imunológicos. Rev Bras Med Esporte. 2006;12(1):21-6. 